\title{
Interactions between cancer stem cells and their niche govern metastatic colonization
}

\author{
Ilaria Malanchi ${ }^{1 *}$, Albert Santamaria-Martínez ${ }^{1 *}$, Evelyn Susanto ${ }^{1}$, Hong Peng ${ }^{1,2}$, Hans-Anton Lehr ${ }^{3}$, Jean-Francois Delaloye ${ }^{4}$ \\ \& Joerg Huelsken ${ }^{1}$
}

\begin{abstract}
Metastatic growth in distant organs is the major cause of cancer mortality. The development of metastasis is a multistage process with several rate-limiting steps ${ }^{1}$. Although dissemination of tumour cells seems to be an early and frequent event ${ }^{2}$, the successful initiation of metastatic growth, a process termed 'metastatic colonization', is inefficient for many cancer types and is accomplished only by a minority of cancer cells that reach distant sites ${ }^{3,4}$. Prevalent target sites are characteristic of many tumour entities ${ }^{5}$, suggesting that inadequate support by distant tissues contributes to the inefficiency of the metastatic process. Here we show that a small population of cancer stem cells is critical for metastatic colonization, that is, the initial expansion of cancer cells at the secondary site, and that stromal niche signals are crucial to this expansion process. We find that periostin (POSTN), a component of the extracellular matrix, is expressed by fibroblasts in the normal tissue and in the stroma of the primary tumour. Infiltrating tumour cells need to induce stromal POSTN expression in the secondary target organ (in this case lung) to initiate colonization. POSTN is required to allow cancer stem cell maintenance, and blocking its function prevents metastasis. POSTN recruits Wnt ligands and thereby increases Wnt signalling in cancer stem cells. We suggest that the education of stromal cells by infiltrating tumour cells is an important step in metastatic colonization and that preventing de novo niche formation may be a novel strategy for the treatment of metastatic disease.
\end{abstract}

We aimed to explore limiting factors that determine metastatic success using the MMTV-PyMT mouse breast cancer model, which spontaneously metastasizes to the lungs ${ }^{6}$. We reasoned that the recently identified cancer stem cells (CSCs, also called tumour-initiating cells), a subset of cancer cells that allow long-term tumour growth and are thought to be responsible for remissions ${ }^{7,8}$, might also be relevant to the development of metastatic disease (Supplementary Fig. 1). We measured the relative size of the population of CSCs from primary MMTV-PyMT tumours and their pulmonary metastases using the previously established markers CD90 and CD24, which label a subset of the $\mathrm{CD} 24^{+} \mathrm{CD} 29^{\mathrm{hi}}$ or $\mathrm{CD} 24^{+} \mathrm{CD} 49 \mathrm{f}^{\text {hi }}$ population used earlier to isolate CSCs and normal mammary gland stem cells ${ }^{9-13}$ (Supplementary Fig. 2). This CSC subset accounts for $3 \pm 2.1 \%$ (s.d.) of all tumour cells from both primary tumours and metastases (Fig. 1a). When $\mathrm{CD} 0^{+} \mathrm{CD} 24^{+} \mathrm{CSC}$ or $\mathrm{CD} 90^{+} \mathrm{CD} 24^{+}$-depleted non-CSCs are separately isolated from $\mathrm{GFP}^{+}$tumours and directly introduced into mice through tail vein injection (GFP, green fluorescent protein), only the CSC population is able to produce lung metastases (Fig. 1b). Moreover, $\mathrm{CD} 0^{+} \mathrm{CD} 24^{+}$cells isolated subsequently from pulmonary metastases are again the only tumour cell population that efficiently initiates secondary metastases (Fig. 1c). This is not due to differences in the extravasation capabilities of CSCs and non-CSCs (Supplementary Fig. 3).
In time course experiments, the relative size of the CSC population changes drastically during metastatic colonization. When injecting unfractionated tumour cells, the total number of tumour cells in the lung declines rapidly within the first $7 \mathrm{~d}$ after seeding ${ }^{14}$ (Fig. $1 \mathrm{~d}$ and Supplementary Fig. 4). Notably, the relative amount of CSCs transiently increases within the first and second weeks to more than $20 \%$. This is due to selective expansion of the stem cell population as a result of their proliferation rate increasing over sixfold relative to the primary tumour in this early phase of metastatic colonization (as measured by BrdU incorporation; Fig. 1e and Supplementary Fig. 5). By contrast, non-CSCs show reduced proliferation $7 \mathrm{~d}$ after injection and, notably, fail to generate CSCs (Fig. 1e and Supplementary Fig. 6). Consequently, only CSCs are able to form metastatic colonies, and non-CSCs remain as single cells (Fig. 1f). Together, this demonstrates that selective expansion of CSCs is responsible for the initiation of metastasis.

The number of injected CSCs evidently exceeds the number of metastatic nodules, indicating that additional factors restrict successful metastatic colonization. Stem cells are suspected to rely on signals from their stromal environment, such as localized growth factors that can affect stem cell maintenance and proliferation ${ }^{15-17}$. We identified Postn as a stromal factor of normal stem cell niches and the metastatic niche (Supplementary Methods). Postn encodes for periostin, which becomes incorporated in the extracellular matrix ${ }^{18}$ and has a role in bone, tooth and heart development and function ${ }^{19-21}$. Its expression is downregulated in the adult except in niches in direct contact with tissue-specific stem cells in mammary gland, bone, skin and intestine (Fig. 2a and Supplementary Figs 7 and 8). In tumours, POSTN is produced by stromal $\alpha \mathrm{SMA}^{+} \mathrm{VIM}^{+}$( $\alpha \mathrm{SMA}$ also known as ACTA2) fibroblasts according to RNA in situ hybridization, immunostaining and quantitative PCR (Fig. 2b-h). Notably, POSTN expression is induced in the lung stroma by infiltrating cancer cells (Fig. 2d), but does not occur in the alveolar lung tissue of tumour-bearing but metastasis-free animals (Supplementary Fig. 8). Human breast cancer patients show induction of stromal POSTN expression in $75 \%$ of lymph node metastases (Fig. 2i, j and Supplementary Fig. 9). Whereas POSTNdeficient mice maintain normal mammary gland development (Supplementary Figs 10-12), MMTV-PyMT Postn ${ }^{-1-}$ breast cancers show a dramatic decrease in the number and size of pulmonary metastases, to less than $10 \%$ of controls, despite unaltered primary tumour size and morphology $(n=37, P<0.003$; Fig. 3a-d and Supplementary Figs 1315). Metastasis formation from POSTN-deficient tumour cells is rescued in wild-type recipients (Supplementary Figs 16 and 17), where expression of POSTN is at the same level as in controls (Supplementary Fig. 18), indicating that stromal production of POSTN determines metastatic efficiency.

In searching for tumour-derived factors that can induce stromal POSTN, in silico promoter analysis predicted regulation mediated

${ }^{1}$ Ecole Polytechnique Fédérale de Lausanne, Swiss Institute for Experimental Cancer Research and National Center of Competence in Research 'Molecular Oncology', 1015 Lausanne, Switzerland.

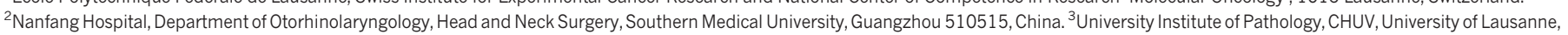
1011 Lausanne, Switzerland. ${ }^{4}$ Department of Gynecology and Obstetrics, Centre Hospitalier Universitaire Vaudois, 1011 Lausanne, Switzerland.

*These authors contributed equally to this work. 

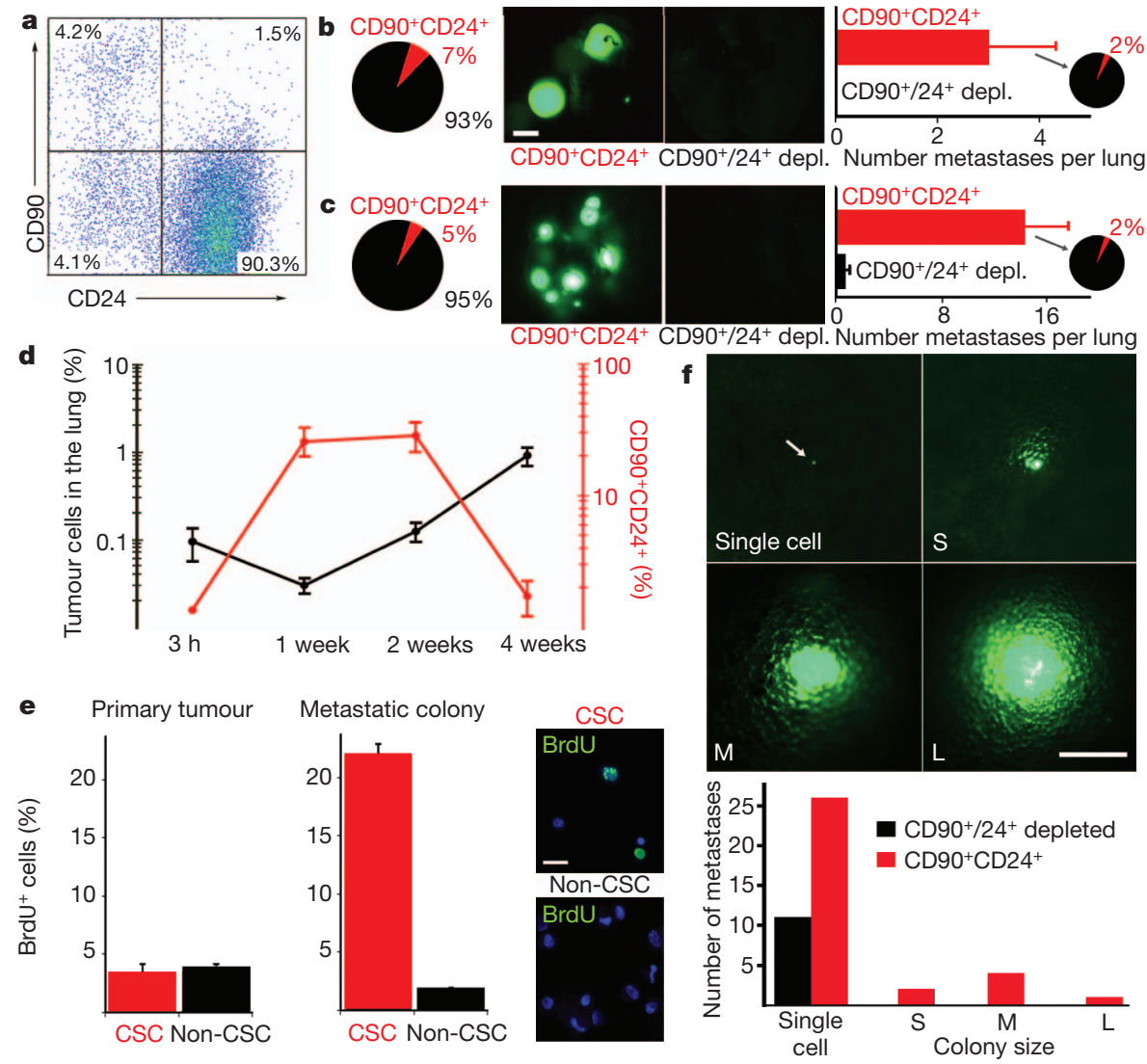

Figure $1 \mid$ Cancer stem cells initiate metastasis. (a) Representative density plot showing the abundance of cancer stem cells in MMTV-PyMT breast tumours defined as $\mathrm{CD} 24^{+} \mathrm{CD} 90^{+}$after gating for viable $\left(7-\mathrm{AAD}^{-}\right)$and lin $\left(\mathrm{CD} 31^{-} \mathrm{CD} 45^{-} \mathrm{TER} 19^{-}\right.$) cells (not shown). b, c, CSCs are the only cells to form pulmonary metastases on tail vein injection. $\mathrm{CD} 24^{+} \mathrm{CD} 90^{+} \mathrm{CSCs}$ and non-CSC populations from $\mathrm{GFP}^{+}$tumour cells freshly isolated from primary tumours $\left(\mathbf{b} ; 10^{4}\right.$ cells injected each) and metastases $\left(\mathbf{c} ; 10^{5}\right.$ cells injected each) were separately injected in recipient mice. The frequency of CSCs (red; $P<0.01$ (b), $P<0.05$ (c)) is maintained at the metastatic site (shown as pie charts; $n=6$ each; errors, s.e.m.). Scale bar, $1 \mathrm{~mm}$. d, Time course experiments show selective proliferation of CSCs during metastatic colonization. GFP ${ }^{+}$tumour cells $\left(10^{6}\right)$ were intravenously injected into recipient mice. At the indicated time points, the total proportion of $\mathrm{GFP}^{+}$tumour cells in the lung (black line) and the relative amount of the CSC population (red line) were evaluated by analysis using fluorescence-activated cell sorting (FACS). Note the transient increase of
CSCs after one week ( $n=4$ per time point; errors, s.e.m.). e, f, CSCs are the only cells able to initiate growth in a short-term in vivo colonization assay. e, One week after tail vein injection of $10^{6} \mathrm{GFP}^{+}$tumour cells, mice were injected with BrdU. After $2 \mathrm{~h}, \mathrm{CSC}$ and non-CSCs were isolated by FACS and the frequency of proliferating cells was evaluated by BrdU staining on cytospins $(n=12$, $P<0.05$; errors, s.d.; representative example is shown on the right). Note the increase of proliferation in the CSC population during the early phase of lung colonization; by contrast, proliferation does not significantly differ between the two populations in the primary tumour. $\mathrm{f}, \mathrm{GFP}^{+} \mathrm{CSCs}\left(2 \times 10^{5}\right)$ or $\mathrm{GFP}^{+}$nonCSCs $\left(4 \times 10^{5}\right)$ were tail vein injected and analysed after two weeks. $\mathrm{CD}^{+} 0^{+} /$ $\mathrm{CD} 24^{+}$-depleted non-CSCs remained as single cells whereas CSCs were able to initiate growth and form metastases of different sizes (S, 30-300 cells; M, 3003,000 cells; $L,>3,000$ cells). The upper panel shows a representative example of the different metastasis colonies and the results are cumulatively quantified in the bottom panel $(n=6)$. Scale bars, $20 \mu \mathrm{m}(\mathbf{e})$ and $250 \mu \mathrm{m}(\mathbf{f})$. by SMAD, NF- $\kappa \mathrm{B}$ and LEF/TCF. Primary lung fibroblasts upregulate POSTN in response to TGF- $\beta 3$ and TGF- $\beta 2$, but are not responsive to BMP4, Wnt3A, Wnt5A or TNF- $\alpha$ (Fig. 3e, Supplementary Fig. 19 and data not shown). Co-culture experiments revealed that tumour cells are sufficient to trigger POSTN production (Supplementary Fig. 20). Notably, CSCs and non-CSCs both produce high levels of TGF- $\beta 3$ (Supplementary Fig. 21), and blocking the action of TGF- $\beta 3$ by expression of a secreted decoy receptor ${ }^{22}$ (TGF $\beta$ R2 $\Delta$ TM; see Methods) in tumour cells blocks POSTN expression and prevents metastasis formation (Fig. 3f and Supplementary Fig. 22) in line with earlier results ${ }^{23}$. Together, these experiments demonstrate that infiltrating tumour cells need to educate the host stroma of the target organ to support metastasis initiation.

Even small metastatic colonies are strongly diminished in POSTNdeficient animals (Supplementary Fig. 23). Having shown that metastatic colonization depends on cancer stem cells, we went on to assess a potential role of POSTN in stem cell maintenance. Growth under conditions of ultralow attachment has been used to study CSCs in vitro ${ }^{24}$. Surprisingly, establishment of such cancer stem cell cultures is not possible using POSTN-deficient tumours (Fig. $3 \mathrm{~g}-\mathrm{j} ; n=16$; compare with Supplementary Fig. 24, which shows unaltered cell survival in standard two-dimensional culture). In wild-type tumour spheres, POSTN is expressed by tumour-derived, stromal fibroblasts (Supplementary Fig. 25). Adding periostin protein to the mutant tumour cells rescues sphere formation (Fig. 3j). Conversely, a blocking antibody targeting POSTN prevents maintenance of wild-type cancer stem cells (Supplementary Fig. 30), but affects neither cellular survival nor growth per se (Supplementary Figs 26 and 27). Furthermore, CSCs fail to proliferate when co-cultured with POSTN-mutant, pulmonary fibroblasts (Fig. 3k). In lung metastases, we observe a preferential localization of $\mathrm{CD}^{+}{ }^{+} \mathrm{CSCs}$ adjacent to stromal niches (Fig. 31), whereas lung metastases in POSTN-deficient animals show a reduction in the size of the CSC population (Fig. $3 \mathrm{~m}$ ). Thus, POSTN is an essential niche factor that supports CSC growth during metastatic colonization.

To gain insight into how POSTN is related to the mechanisms that control stem cell maintenance, we characterized the interactome of POSTN by tandem affinity purification (TAP)-tag enrichment and 


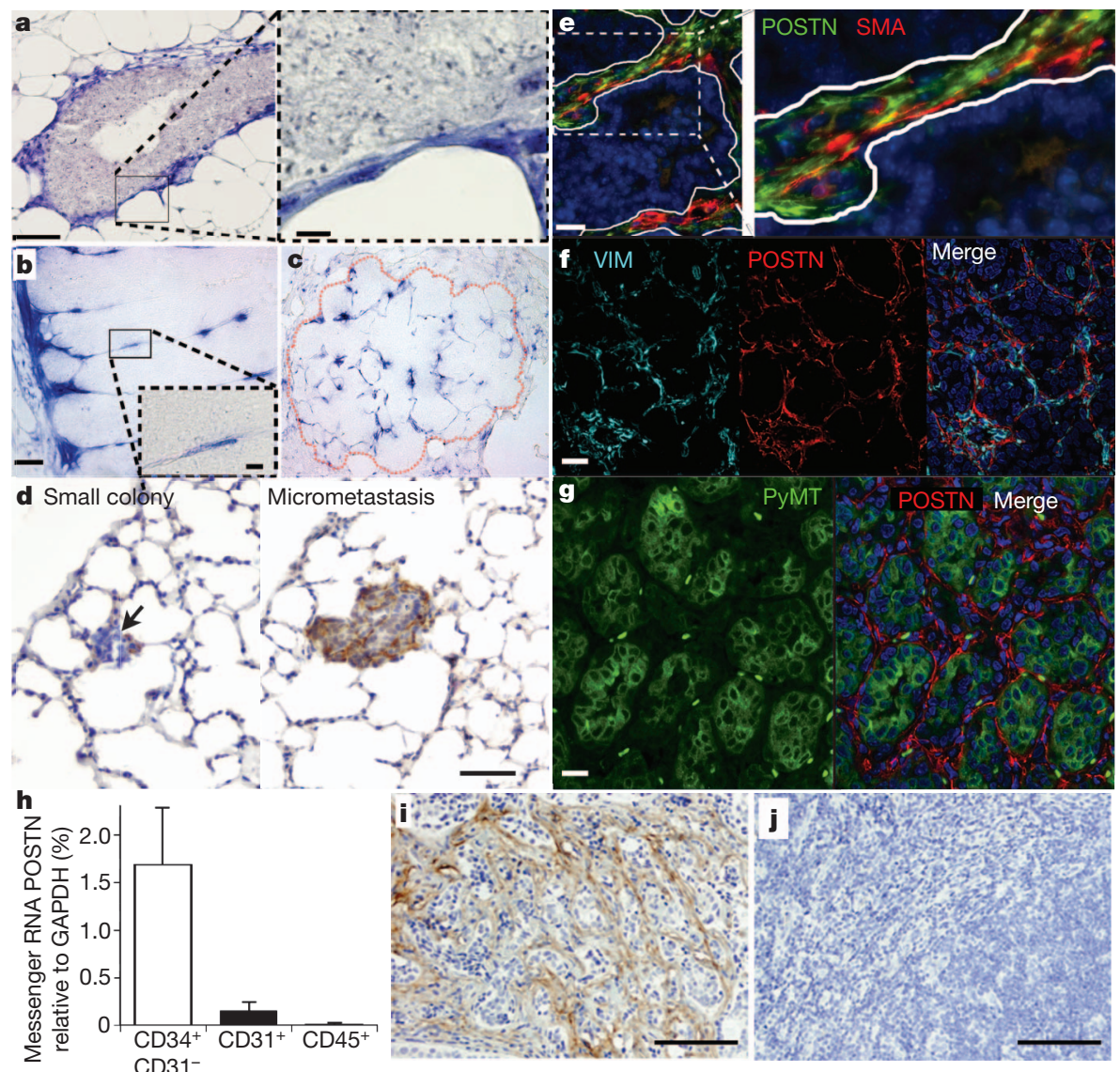

Figure 2 POSTN is a stromal niche component that is induced on metastasis formation. a-c, Stromal POSTN expression as detected by RNA in situ hybridization (POSTN-expressing cells in blue) around terminal end buds, which are enriched for mammary gland stem cells during puberty (a), tumour cells at the primary site (b) and in pulmonary metastases (c; outlined in red). d, Immunohistochemistry of POSTN expression in pulmonary metastases at different stages. $\mathbf{e}-\mathbf{g}$, Immunofluorescence analysis of POSTN and fibroblast markers in pulmonary metastases. POSTN is not expressed by PyMT tumour cells (g), but is from $\alpha \mathrm{SMA}^{+}$(e) and $\mathrm{VIM}^{+}$(f) fibroblasts. The white outline in

tandem mass spectrometry (Supplementary Methods), and this pointed to binding of POSTN to Wnt ligands ${ }^{25}$. Using TAP-tag pull-downs, we confirmed interaction between POSTN and Wntl and Wnt3A, but found that a known inhibitory ligand of the pathway, DKK1, does not associate with POSTN (Fig. 4a, b and Supplementary Fig. 28). The interaction between POSTN and Wnt ligands boosts Wnt signalling activity as measured by LRP6 phosphorylation and reporter assays using Wnt1, Wnt2 and Wnt6 (Fig. 4c, d). The Wnt pathway is known to control stem cell maintenance in a variety of tissues, including mammary gland ${ }^{26}$ and tumours ${ }^{8,27,28}$. Indeed, addition of Wnt3A can rescue in vitro stem cell expansion in the absence of POSTN (Supplementary Figs 30 and 31). Furthermore, we find Wnt signalling activity to be concentrated in the CSC population in vivo as analysed by FACS (Fig. 4e, f). Here, we use a lentiviral reporter that allows Wntinducible GFP expression and constitutive labelling of individual reporter-containing cells by human CD2 (Supplementary Fig. 29). Wnt signalling activity in metastases is abrogated in the absence of POSTN (Fig. 4g; see Supplementary Fig. 29 for Wnt activity in tumour spheres). Similarly, we detect higher levels of the general Wnt target gene Axin 2 in metastases in wild-type hosts than in mutant hosts (Fig. 4h, i). Remarkably, metastasis of Wnt-driven breast cancer proves to be independent of stromal POSTN (Fig. 4j). Thus, POSTN acts as a niche component that can promote stem cell maintenance and metastatic colonization by augmenting Wnt signalling (Supplementary Fig. 1). e defines the tumour-stroma border. h, Quantitative PCR with reverse transcription detects POSTN expression in $\mathrm{CD} 34^{+} \mathrm{CD} 31^{-}$pulmonary fibroblasts, in $\mathrm{CD}_{3} 1^{+}$endothelial cells but not in $\mathrm{CD} 45^{+}$immune cells that were isolated by FACS from lungs of mice with macrometastases $(n=3$; errors s.d.). $\mathbf{i}, \mathbf{j}$, Immunohistochemistry of POSTN expression in auxiliary lymph nodes of human breast cancer patients with metastasis-free N0 disease (j) or metastatic disease (i). A total of 17 of 23 lymph node metastases were positive for POSTN expression. Scale bars: $20 \mu \mathrm{m}$ (a) and $2.5 \mu \mathrm{m}$ (a, inset); $50 \mu \mathrm{m}$ (b; same scale in c) and $10 \mu \mathrm{m}(\mathbf{b}$, inset); $50 \mu \mathrm{m}(\mathbf{d}) ; 20 \mu \mathrm{m}(\mathbf{e}-\mathbf{g}) ; 200 \mu \mathrm{m}(\mathbf{i}, \mathbf{j})$.
Thus, instrumental factors that contribute to the inefficiency of the metastatic process are the low abundance of CSCs, which are required to initiate growth at the secondary site, and incompatibilities with 'foreign' niches, which necessitate education of the target organ to resemble the primary microenvironment. Periostin is an essential component of this CSC-supportive niche and needs to be induced in the lung stroma by infiltrating tumour cells. Together with a recent report that implicated tumour-cell-derived tenascin $\mathrm{C}$ in breast cancer metastasis, these examples demonstrate an essential role for single proteins of the extracellular matrix in metastatic colonization ${ }^{29,30}$.

Surprisingly, POSTN deficiency affects neither normal mammary gland development nor tumour formation or the relative size of the CSC population at the primary site (Supplementary Fig. 32). This may be due to a greater complexity and redundancy in the primary niche, such that the loss of a single factor can be tolerated. By contrast, loss of only one important factor, such as POSTN, prevents metastatic colonization in the presumably less supportive and less complex secondary site. Accordingly, the early phase of metastasis can be anticipated to be particularly sensitive to therapeutic intervention because the dependence of cancer cells on niche signals is probably highest in that phase. Targeting this metastatic niche promises to be less sensitive to rapid genetic changes in cancer cells and may not only prevent metastatic colonization but may also interfere with the survival of disseminated, dormant cancer cells. 

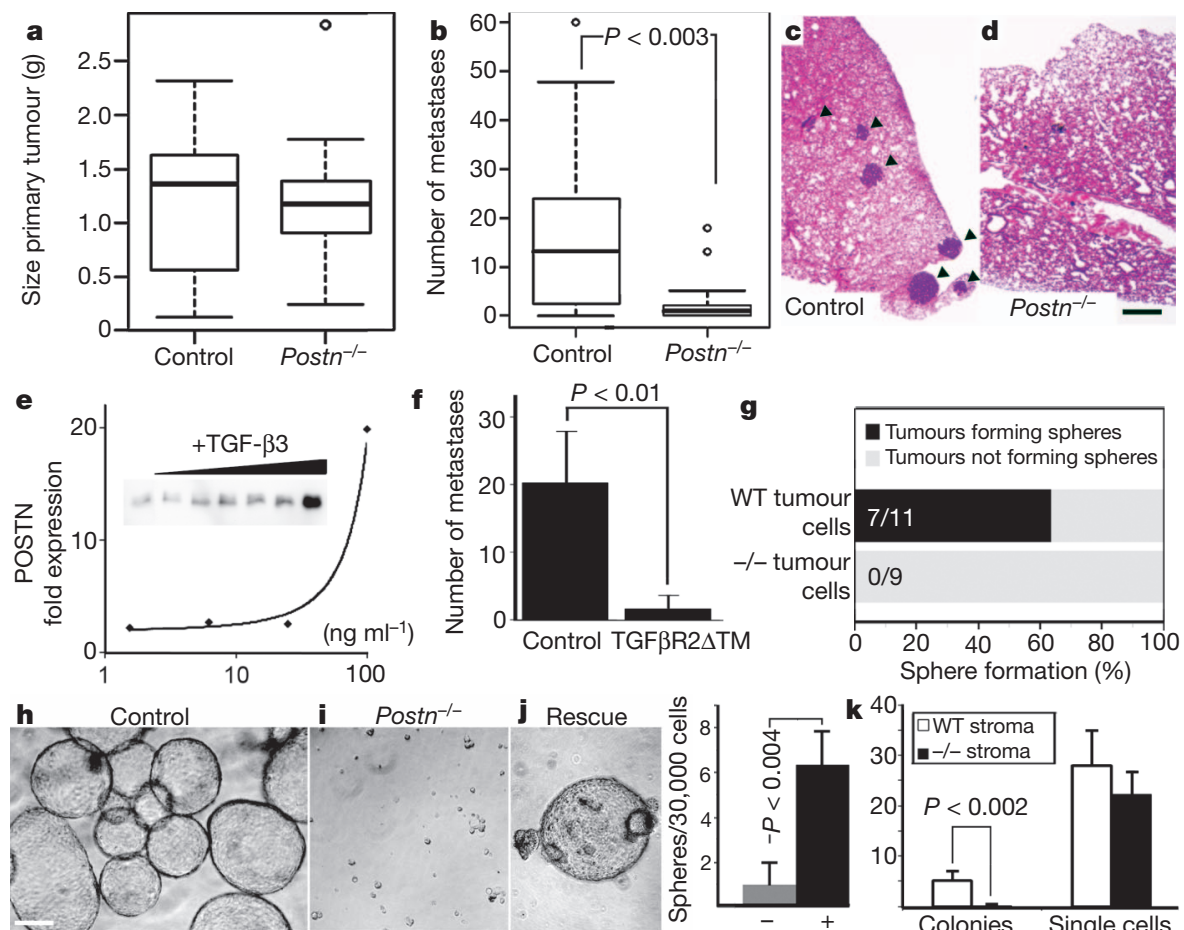

${ }^{40}$
30
20
10

Sphere formation (\%)
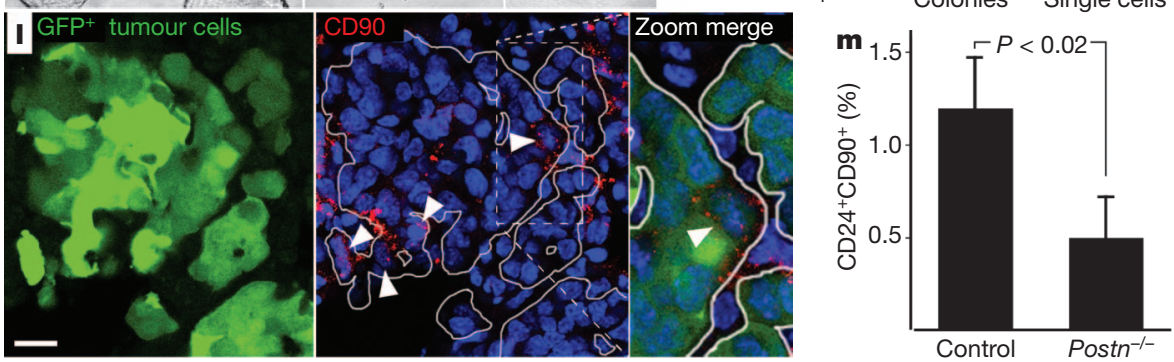

Figure $3 \mid$ POSTN is required for metastatic colonization by affecting CSC maintenance. $\mathbf{a}, \mathbf{b}$, Primary tumour size (a) and number of spontaneously formed macrometastases (b) in control and Postn ${ }^{-1-}$ mice $(n=37) . \mathbf{c}, \mathbf{d}$, HE section of spontaneous, pulmonary metastases in control (c) and POSTN-mutant (d) animals. e-f, TGF- $\beta 3$ induces POSTN expression in lung stromal cells. e, TGF- $\beta 3$ was added to lung stromal cells to test its ability to induce POSTN expression. RNA expression by quantitative PCR and protein expression by western blot analysis (inset) were measured at the indicated concentrations. f, Blocking of TGF- $\beta 3$ abrogates metastasis formation. Cancer cells were infected either with a control virus or with a virus expressing secreted, dominant negative TGF $\beta R 2 \Delta T M$ and separately intravenously injected into recipient mice. Metastasis formation was evaluated 6 weeks after injection $(n=12$; errors, s.d.). g, Frequency of mammosphere formation from primary tumours. WT, wild type. h, Representative picture of control tumour mammospheres. i, Loss of CSCs in secondary mammosphere assays from Postn ${ }^{-1-}$ tumours. $\mathbf{j}$, Sphere formation by Postn ${ }^{-1-}$ tumour cells can be rescued by addition of $50 \mathrm{ng} \mathrm{ml}^{-1}$ of periostin to primary cultures. Control tumour cells produce $13.3 \pm 2.5$ spheres per 30,000 cells. Quantification is shown in the histogram on the right ( $n=4$; errors, s.d.). $\mathbf{k}$, Only stromal cells isolated from wild-type lungs are able to support CSC colony formation in vitro. CSCs were sorted by FACS and seeded on lung stromal cells isolated either from wild-type or Postn ${ }^{-1-}$ mice. Colony formation was evaluated after two weeks ( $n=3$; errors, s.d.).

l, Immunofluorescence analysis shows that $\mathrm{GFP}^{+} \mathrm{CD}^{+} 0^{+} \mathrm{CSC}$ (white arrow heads) are concentrated in areas with immediate contact to the stroma. m, Reduced frequency of $\mathrm{CD} 24^{+} \mathrm{CD} 90^{+}$ CSCs in rare pulmonary metastases in POSTNdeficient mice ( $n=12$; errors, s.d.). Scale bars: $500 \mu \mathrm{m}(\mathbf{c}, \mathbf{d}) ; 50 \mu \mathrm{m}(\mathbf{h}-\mathbf{j}) ; 5 \mu \mathrm{m}(\mathbf{l})$.
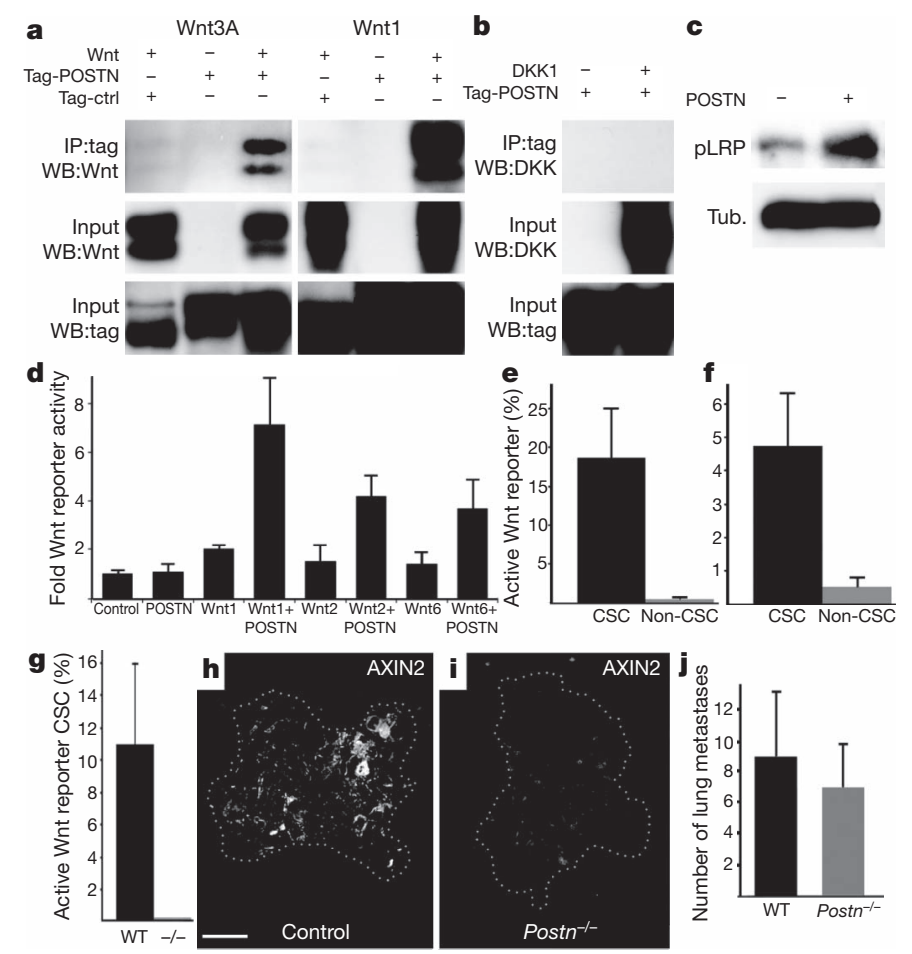

Figure $4 \mid$ POSTN promotes stem cell maintenance and metastasis

formation by augmenting Wnt signalling. a, Interaction of TAP-tagged POSTN and Wnt3A and Wnt 1 as determined by pull down and western blotting. $\mathbf{b}$, Interaction of TAP-tagged POSTN and DKK1 as determined by pull down and western blotting. Note the absence of interaction between POSTN and DKK1. c, LRP6 phosphorylation (pLRP) increases on POSTN expression. d, Wnt reporter activity in response to Wnt1, Wnt2 or Wnt6 is stimulated by POSTN ( $n=3$; errors, s.d.). e, f, In vivo Wnt reporter assay. Tumour cells were infected with a lentivirus containing both an artificial Wntresponsive promoter driving GFP expression and a constitutive promoter expressing human CD2 (Supplementary Fig. 29). Cells were analysed by FACS considering only human-CD2 ${ }^{+}$, reporter-containing cells. $\mathrm{CD} 90^{+} \mathrm{CD} 24^{+}$ CSCs and non-CSCs were scored for Wnt reporter activity as GFP ${ }^{+}$. The signal concentrates in CSCs in both primary tumours (e) and pulmonary metastases (f) $\left(n=3\right.$; errors, s.e.m.). g, CD $90^{+} \mathrm{CD} 24^{+}$wild-type CSCs containing a Wnt reporter (human $\mathrm{CD}^{+}$) show signalling activity $\left(\mathrm{GFP}^{+}\right)$only when seeded in wild-type as opposed to Postn ${ }^{-1-}$ lungs ( $n=3$; errors, s.e.m.).

h, i, Immunofluorescence staining of AXIN2 in control (h) and POSTNdeficient (i) lung metastases (outlined in white). Note the absence of staining in POSTN $^{-}$lung metastases. Scale bar, $10 \mu \mathrm{m}$. j, MMTV-Wnt1 tumour cells can form pulmonary metastases in the absence of POSTN expression in the stroma. Tumour cells were orthotopically transplanted into mammary glands of wildtype or POSTN-deficient recipients and analysed after ten weeks for spontaneous lung metastasis ( $n=5$ each; errors, s.d.). 


\section{METHODS SUMMARY}

Details on the mouse models used and the generation of the Postn gene ablation can be found in Supplementary Information. Tumours derived from MMTVPyMT mice were collected and cells were isolated and directly stained for FACS analysis or sorting using antibodies against CD31, CD45, TER119 (LY76), CD90, CD24 and CD49f (ITGA6). FACS-isolated tumour cells were directly transplanted into recipient mice.

Full Methods and any associated references are available in the online version of the paper at www.nature.com/nature.

Received 26 July 2010; accepted 1 November 2011.

Published online 7 December 2011.

1. Chambers, A. F., Groom, A. C. \& MacDonald, I. C. Dissemination and growth of cancer cells in metastatic sites. Nature Rev. Cancer 2, 563-572 (2002).

2. Hüsemann, Y. et al. Systemic spread is an early step in breast cancer. Cancer Cell 13, 58-68 (2008).

3. Kouros-Mehr, H. et al. GATA-3 links tumor differentiation and dissemination in a luminal breast cancer model. Cancer Cell 13, 141-152 (2008).

4. Nguyen, D. X., Bos, P. D. \& Massague, J. Metastasis: from dissemination to organspecific colonization. Nature Rev. Cancer 9, 274-284 (2009).

5. Hess, K. R. et al. Metastatic patterns in adenocarcinoma. Cancer 106, 1624-1633 (2006).

6. Lin, E. Y. et al. Progression to malignancy in the polyoma middle T oncoprotein mouse breast cancer model provides a reliable model for human diseases. Am. J. Pathol. 163, 2113-2126 (2003)

7. Al-Hajj, M., Wicha, M. S., Benito-Hernandez, A., Morrison, S. J. \& Clarke, M. F. Prospective identification of tumorigenic breast cancer cells. Proc. Natl Acad. Sci. USA 100, 3983-3988 (2003).

8. Reya, T.\& Clevers, H. Wnt signalling in stem cells and cancer. Nature $\mathbf{4 3 4 , 8 4 3 - 8 5 0}$ (2005).

9. Cho, R. W. et al. Isolation and molecular characterization of cancer stem cells in MMTV-Wnt-1 murine breast tumors. Stem Cells 26, 364-371 (2008).

10. Shackleton, M. etal. Generation of a functional mammary gland from a single stem cell. Nature 439, 84-88 (2006).

11. Stingl,J. etal. Purification and unique properties of mammary epithelial stem cells. Nature 439, 993-997 (2006).

12. Liu, J. C., Deng, T., Lehal, R. S., Kim, J. \& Zacksenhaus, E. Identification of tumorsphere-and tumor-initiating cells in HER2/Neu-induced mammary tumors. Cancer Res. 67, 8671-8681 (2007).

13. Zhang, M. etal. Identification of tumor-initiating cells in a p53-null mouse model of breast cancer. Cancer Res. 68, 4674-4682 (2008).

14. Podsypanina, K. et al. Seeding and propagation of untransformed mouse mammary cells in the lung. Science 321, 1841-1844 (2008).

15. Vermeulen, L. etal. Wnt activity defines colon cancer stem cells and is regulated by the microenvironment. Nature Cell Biol. 12, 468-476 (2010).

16. Visvader, J. E. \& Lindeman, G. J. Cancer stem cells in solid tumours: accumulating evidence and unresolved questions. Nature Rev. Cancer 8, 755-768 (2008).
17. Psaila, B. \& Lyden, D. The metastatic niche: adapting the foreign soil. Nature Rev. Cancer 9, 285-293 (2009).

18. Takeshita, S., Kikuno, R., Tezuka, K. \& Amann, E. Osteoblast-specific factor 2: cloning of a putative bone adhesion protein with homology with the insect protein fasciclin I. Biochem. J. 294, 271-278 (1993).

19. Shimazaki, M. \& Kudo, A. Impaired capsule formation of tumors in periostin-null mice. Biochem. Biophys. Res. Commun. 367, 736-742 (2008).

20. Oka, T. et al. Genetic manipulation of periostin expression reveals a role in cardiac hypertrophy and ventricular remodeling. Circ. Res. 101, 313-321 (2007).

21. Rios, H. et al. Periostin null mice exhibit dwarfism, incisor enamel defects, and an early-onset periodontal disease-like phenotype. Mol. Cell. Biol. 25, 11131-11144 (2005).

22. Zhao, W. etal. Suppression of in vivo tumorigenicity of rat hepatoma cell line KDH-8 cells by soluble TGF-beta receptor type II. Cancer Immunol. Immunother. 51, 381-388 (2002).

23. Muraoka, R. S. et al. Blockade of TGF-beta inhibits mammary tumor cell viability, migration, and metastases. J. Clin. Invest. 109, 1551-1559 (2002).

24. Pece, S. et al. Biological and molecular heterogeneity of breast cancers correlates with their cancer stem cell content. Cell 140, 62-73 (2010).

25. Milovanovic, T. et al. Expression of Wnt genes and frizzled 1 and 2 receptors in normal breast epithelium and infiltrating breast carcinoma. Int. J. Oncol. 25, 1337-1342 (2004)

26. Zeng, Y. A. \& Nusse, R. Wnt proteins are self-renewal factors for mammary stem cells and promote their long-term expansion in culture. Cell Stem Cell 6, 568-577 (2010).

27. Malanchi, I. et al. Cutaneous cancer stem cell maintenance is dependent on $\beta$-catenin signaling. Nature 452, 650-653 (2008).

28. Barker, N. et al. Crypt stem cells as the cells-of-origin of intestinal cancer. Nature 457, 608-611 (2009).

29. Wels, J., Kaplan, R. N., Rafii, S. \& Lyden, D. Migratory neighbors and distant invaders: tumor-associated niche cells. Genes Dev. 22, 559-574 (2008).

30. Oskarsson, T. et al. Breast cancer cells produce tenascin $\mathrm{C}$ as a metastatic niche component to colonize the lungs. Nature Med. 17, 867-874 (2011).

Supplementary Information is linked to the online version of the paper at www.nature.com/nature.

Acknowledgements We are grateful to U. Koch for advice on FACS, to M. Moniatte for advice on mass spectrometry and to S. Leuba for technical assistance with histology. I.M., E.S., A.S.-M. and J.H. were supported in part by the Swiss League against Cancer, the SNF, the NCCR in Molecular Oncology and the Anna Fuller Fund. J.H. holds the EPFL chair for Signal Transduction in Oncogenesis sponsored by Debiopharm.

Author Contributions I.M., A.S.-M. and J.H. designed and performed most of the experiments, analysed data and prepared the manuscript; E.S. and H.P. performed experiments; H.-A.L. performed experiments and analysed data; J.-F.D. provided clinical samples; and J.H. designed and supervised the study.

Author Information Reprints and permissions information is available at www.nature.com/reprints. The authors declare no competing financial interests. Readers are welcome to comment on the online version of this article at www.nature.com/nature. Correspondence and requests for materials should be addressed to J.H. (joerg.huelsken@epfl.ch). 


\section{METHODS}

Mouse work. The design of the POSTN targeting vector is shown in Supplementary Fig. 10. E14.1 129/Ola ES cells were used for targeting and were injected into C57Bl6 blastocysts. The POSTN mutant allele was then backcrossed onto the FVB background for at least eight generations. The MMTV-PyMT ${ }^{31}$, MMTV-Wnt ${ }^{32}$ and the ACT-GFP ${ }^{33}$ mouse strains have been described previously. All used strains were backcrossed onto FVB for ten generations except for the Nude allele, which was used on a NMRI background. For tumour cell transplantations, Nude or Rag1 ${ }^{-1-}$ mice were used when tumour cells expressed GFP or had been modified by lentiviral vectors. In general, we did not observe major differences in these different strains with respect to cancer stem cell phenotype, tumour take rate or metastasis number, except for an overall faster growth in $\mathrm{Ragl}^{-/-}$versus Nude versus wild type. For tail vein injections, tumour cells were resuspended in $100 \mu \mathrm{l}$ of PBS. For orthotopic transplantations, tumour cells were resuspended in $50 \mu$ l growth-factor-reduced matrigel (BD Biosciences) and transplanted into a small pocket within the fourth mammary fat pad. All animal procedures were performed in accordance with the Swiss legislation on animal experimentation.

Antibodies. We used TER119, CD24, CD29, CD34, CD45 and CD90.1 (eBioscience); CD31 and GFP (Invitrogen); Cytokeratin 8, POSTN, AXIN2 and Wnt1 (Abcam); POSTN (Adipogen); Cytokeratin 14 (Covance); GATA-3 and PyMT (Santa Cruz Biotechnology); $\alpha$ SMA (Sigma); VIM (Lifespan Biosciences); and BrdU (BD Pharmingen and ImmunologicalsDirect). The antibody against human CD2 was a kind gift by Professor Werner Held (Ludwig Center for Cancer Research, Lausanne).

Tumour and metastasis evaluation. For the quantification of spontaneous micrometastasis, lungs were completely sectioned and HE stained, and the size and the number of metastatic nodules were analysed by microscopy. Quantification of lung metastases derived from $\mathrm{GFP}^{+}$tumour cells was performed by fluorescent microscopy (Leica M205 FA stereomicroscope) or by FACS analysis of cell preparations from total lungs. All statistical evaluation used a homoscedastic Student's $t$-test with a two-tailed distribution.

For expression of a secreted TGF $\beta$ decoy receptor (TGF $\beta$ R $2 \Delta$ TM), tumour cells were transduced with lentiviral vectors expressing the extracellular domain (amino acids 1-185) of murine TGFßR2 (NM_009371.3) under the control of a murine phosphoglycerate kinase (PGK) promoter, and metastases were scored as described above following tail vein injection of transduced tumour cells.

Tumour cell isolation. Tumours or lungs containing pulmonary metastases were minced with a razor blade and digested with a mixture of DNase and Liberase (Roche Diagnostics). On enzymatic digestion, samples were passed through a $100-\mu \mathrm{m}$ filter and washed once in growth medium (DMEM/F12 with $2 \%$ FBS $20 \mathrm{ng} \mathrm{ml}^{-1} \mathrm{EGF}, 10 \mu \mathrm{g} \mathrm{ml}^{-1}$ insulin; Invitrogen) and twice in PBS. Cells were then directly used for FACS staining and analysed in a Cyan ADP flow cytometer (Beckman Coulter) or sorted with a custom-built FACSAria II (Beckton Dickinson). For tail vein injection, cells were plated overnight on collagen. In the experiments evaluating the metastasis initiation potential of CSCs versus non-CSCs, we were able to prepare and inject higher cell numbers from metastases than from primary tumours, where larger necrotic areas limit the obtainable cell amounts. In both settings, only CSCs were able to give rise to pulmonary metastases, but this experimental setting is unsuitable for a direct comparison of the metastatic potential of CSCs isolated from primary tumours with that of those isolated from metastases. In the extravasation experiments, we performed several pilot experiments perfusing the lungs before dissection to distinguish cells trapped inside capillaries from those that successfully extravasated. Perfusion was done with Ringer's buffer through the right ventricle for 5-10 min.

Tumour mammosphere cultures. Sphere cultures were established from total tumour cell preparations. After dissociation, tumour cells were plated on collagen overnight, trypsinized and plated in $100 \mu \mathrm{l}$ of sphere media (DMEM/F12 with B27, $20 \mathrm{ng} \mathrm{ml}^{-1} \mathrm{EGF}, 20 \mathrm{ng} \mathrm{ml}^{-1} \mathrm{FGF}$ and $4 \mu \mathrm{g} \mathrm{ml}^{-1}$ heparin) into 96-well, low-attachment plates (Constar) at a concentration of $(1-3) \times 10^{4}$ cells per well. For the antiPOSTN blocking antibody assay, half of the culture medium was replaced by hybridoma supernatant. Secondary mammosphere culture was performed by collecting the spheres through gentle centrifugation $(130 g)$ followed by enzymatic $\left(10 \mathrm{~min}\right.$ in $0.05 \%$ trypsin-EDTA at $37^{\circ} \mathrm{C}$ ) and mechanical dissociation using a $25 \mathrm{G}$ needle. Immunostainings were performed as whole mounts of PFA-fixed and $0.1 \%$ Triton-X100-permeabilized spheres and were analysed by confocal microscopy. Identification of POSTN as a niche-expressed factor. To identify nicheexpressed factors, we performed microarray RNA profiling studies where we analysed laser-capture microdissected material from normal stem cells together with their adjacent stroma. This was compared with laser-capture microdissected material isolated from differentiated cells together with their adjacent stroma from the same tissue (data not shown). Skin and intestine contain stem cells in morphologically distinct structures (the bulge in the skin, the crypt in the intestine). On the basis of these profiles (accessible through GEO GSE31730 and 8818), we evaluated potential niche localization of candidate genes by RNA in situ hybridization and immunofluorescence analysis (Fig. 2a, Supplementary Fig. 7 and data not shown). Moreover, we profiled the candidate genes by quantitative PCR with reverse transcription and performed RNA in situ hybridization in other tissues, in tumours and in metastases (data not shown). This identified POSTN as a stromal marker in primary murine breast cancer and its metastases (Fig. 2).

Immunodetection and in situ hybridization. Immunostaining was performed on 7- $\mu \mathrm{m}$ OCT- or gelatin-embedded (to maintain GFP signals) frozen sections permeabilized with $0.1 \%$ Triton-X100 or on $5-\mu \mathrm{m}$ paraffin sections using antigen retrieval for $20 \mathrm{~min}$ in boiling, $10 \mathrm{mM}$ citric acid, $\mathrm{pH}$ 6.0. For BrdU immunostaining, an additional incubation period of $10 \mathrm{~min}$ in $2 \mathrm{~N} \mathrm{HCl}$ was performed. For immunohistochemistry, endogenous peroxidases were blocked by incubation with $0.6 \% \mathrm{H}_{2} \mathrm{O}_{2}$ in methanol. After incubation with the indicated antibodies, secondary fluorescently labelled antibodies Alexa Fluor 488, 567 and 647 (Molecular Probes, Invitrogen) or HRP-conjugated secondary antibodies were used to reveal the primary antibodies. For immunohistochemistry, Dako Envision ${ }^{+}$was used together with diaminobenzidine tablets (Sigma) to detect HRP. Fluorescent images were made with an automated upright microscope system (DM5500, Leica) or an LSM700 upright or inverted confocal microscope (Zeiss). Light images were made with an AX70 widefield microscope (Olympus). Figures were generated with PHOTOSHOP (Adobe Systems) and CYTOSKETCH (CytoCode).

In situ hybridizations were performed on paraffin sections permeabilized by proteinase K digestion using DIG-labelled (Roche), antisense transcripts of the mouse POSTN complementary DNA. After hybridization, section were washed in $50 \%$ formamide, $\times 5$ SSC $(0.75 \mathrm{M} \mathrm{NaCl}, 85 \mathrm{mM}$ sodium citrate, $\mathrm{pH} 7.0)$ and $0.1 \%$ Tween at $70{ }^{\circ} \mathrm{C}$, and non-specifically bound probe was digested by RnaseA treatment, followed by extensive washing in $50 \%$ formamide, $\times 2$ SSC and $0.1 \%$ Tween at $65^{\circ} \mathrm{C}$. The DIG label was detected by an anti-DIG Fab (Roche) coupled to alkaline phosphatase using FAST NBT/BCIP (Sigma).

Western blot. Protein was extracted with complete RIPA buffer, separated by electrophoresis, transferred to PVDF membranes, blocked with 5\% dry milk or BSA and incubated overnight with primary antibodies. Immunoreactive bands were visualized using HRP-conjugated secondary antibodies (Promega) and the detection reagent SuperSignal West Cheminoluminiscent Substrate (Thermo Scientific).

Real-time RT-PCR. RNA was prepared using a miniRNA or microRNA kit (Qiagen) from FACS-isolated primary cells. Complementary DNAs were generated using oligo-T priming and quantitative PCR was performed in a LightCycler (Roche) or a StepOnePlus thermocycler (Applied Biosystems) using the Power SYBR green PCR Master Mix (Applied Biosystems) and the primers aatgctgccetggc tatatg and gcatgaccettttccttcaa (POSTN); agcgcagacacaacccatag and gggttctgccca catagtacaag (TGF- $\beta 3$ ); and caagctcatttcctggtatgacaat and gttgggatagggectctcttg (GAPDH).

Periostin monoclonal antibody production. In collaboration with Adipogen, we injected Postn ${ }^{-1-}$ mice with purified human POSTN protein. Hybridomas were generated and tested for antibody production by ELISA. Antibodies were selected for their ability to recognize POSTN and for their ability to block tumour mammosphere formation in vitro.

Periostin interactome and pull-downs. Freshly isolated tumour cells were grown under mammosphere conditions in the presence of purified, polyHis-tagged and streptavidin binding peptide (SBP)-tagged POSTN protein (Supplementary Fig. 28) for $72 \mathrm{~h}$. Then proteins were crosslinked by $1 \mathrm{mM}$ DTSSP (Pierce) for $10 \mathrm{~min}$ and solubilized by DDM and SDS (Anatrace). Protein purification was achieved by two successive affinity purification steps using the SBP and the polyHis tags and elution by biotin and imidazole, respectively. After elution, the crosslink was released by DTT treatment, the resulting protein mixture was trypsin-digested and peptides were identified by one-dimensional nano-liquid chromatography tandem mass spectrometry. Among the POSTN-attached proteins, we found Wnt6 to be a potential interactor. Because these experiments involved crosslinkers, we consider these results to be preliminary unless confirmed by independent experiments. For Wnt6, an interaction with POSTN could not be validated owing to a lack of Wnt6 antibodies and a suitable expression system for Wnt6. Nevertheless, this preliminary finding prompted us to test directly for interactions of POSTN with other Wnt ligands where the necessary tools are available. For these pull-downs, cDNAs encoding Wnt 1, HA-tagged Wnt3A, DKK1 and SBP/His-tagged POSTN (Supplementary Fig. 28) or SBP/His-tagged mouse immunoglobulin-G heavy chain (as a control) were transfected separately into 293T cells. Cells secreting POSTN or immunoglobulin-G and cells producing Wnt or DKK were mixed after one day and collected for pull-downs after three additional days. Without the use of crosslinkers, POSTN-interacting proteins were enriched by the SBP/His tag as described above using NP40 as detergent. Western blot was performed for identification of the pulled-down proteins. 
Wnt reporter activity. HEK293T cells were transduced with a lentiviral vector containing a LEF/TCF-responsive luciferase reporter consisting of a minimal promoter and 20 copies of the LEF/TCF consensus site or a control construct in which the LEF/TCF sites had been mutated ${ }^{34}$. These stable Wnt reporter cell lines were then transiently transfected with a POSTN expression vector before these cells were mixed with transiently transfected HEK293T or COS7 cells (the latter for Wnt6 expression) producing Wnt1, Wnt 2 or Wnt6, respectively. The media on these co-cultures of Wnt reporter and Wnt producer cells were replaced after $24 \mathrm{~h}$ to remove soluble Wnt ligands. After $48 \mathrm{~h}$ of co-culture, luciferase activity in cell extracts was measured with a kit from Promega.

For in vivo reporter assays, we generated a lentiviral vector containing the same transcription factor sites and minimal promoter as described above driving GFP expression. In addition, this vector contained a ubiquitous PGK promoter driving expression of a truncated human $\mathrm{CD} 2 \mathrm{cDNA}$ that allows identification of reportercontaining cells by FACS (Supplementary Fig. 29). Lentiviruses were generated in $293 \mathrm{~T}$ cells using third-generation lentivirus packaging vectors, and virus particles were concentrated by ultracentrifugation. Freshly isolated tumour cells were infected with lentiviruses overnight, enriched by anti-CD2 MACS (Miltenyi) for cells carrying the reporter construct and orthotopically transplanted for tumour formation and spontaneous metastasis. Wnt signalling activity in cells of primary tumours or spontaneous pulmonary metastases was quantified by FACS analysis by measuring $\mathrm{GFP}^{+}$cells after gating for human- $\mathrm{CD} 2{ }^{+}$cells. Similarly, human-CD2 ${ }^{+}$ tumour cells were used for tumour mammosphere cultures to measure Wnt pathway activity in the presence or absence of the POSTN blocking antibody.

Isolation of lung stromal cells. Lungs were minced with a razor blade and digested with a mixture of DNase and Liberase (Roche Diagnostics). On enzymatic digestion, samples were passed through a $100-\mu \mathrm{m}$ filter and washed once in growth medium (DMEM/F12 with $2 \% \mathrm{FBS}, 20 \mathrm{ng} \mathrm{ml}^{-1} \mathrm{EGF}$ and $10 \mu \mathrm{ml}^{-1}$ insulin; Invitrogen) and twice in PBS. Cells were plated onto plates pretreated with collagen and allowed to attach for three days. The medium was then changed to serum-free medium and tumour cells or soluble factors were added to test POSTN induction. POSTN expression was evaluated by western blot, PCR with reverse transcription or immunofluorescence analysis.

31. Guy, C. T., Cardiff, R. D. \& Muller, W. J. Induction of mammary tumours by expression of polyomavirus middle T oncogene: a transgenic mouse model for metastatic disease. Mol. Cell. Biol. 12, 954-961 (1992).

32. Tsukamoto, A. S., Grosschedl, R., Guzman, R. C., Parslow, T. \& Varmus, H. E. Expression of the int-1 gene in transgenic mice is associated with mammary gland hyperplasia and adenocarcinomas in male and female mice. Cell 55, 619-625 (1988).

33. Okabe, M., Ikawa, M., Kominami, K., Nakanishi, T. \& Nishimune, Y. 'Green mice’ as a source of ubiquitous green cells. FEBS Lett. 407, 313-319 (1997).

34. Jeannet, G. et al. Long-term, multilineage hematopoiesis occurs in the combined absence of $\beta$-catenin and $\gamma$-catenin. Blood 111, 142-149 (2008). 